Polymer Journal Vol. 1, No. 3, pp. 356-364 (1970)

\title{
Viscoelastic Properties of Monodisperse Polymers. \\ I. Tensile Creep Studies of $\operatorname{Poly}(\alpha$-Methylstyrene)
}

\author{
Hisashi Odani, Norio Nemoto, Shuji Kitamura, \\ and Michio Kurata \\ Institute for Chemical Research, Kyoto University, \\ Uji, Kyoto 611, Japan. \\ and \\ Mikio TAMURA \\ Department of Industrial Chemistry, Kyoto University, \\ Yoshida, Kyoto 606, Japan. \\ (Received February 19, 1970)
}

\begin{abstract}
Tensile creep measurements were made on seven monodisperse polymer samples of poly( $\alpha$-methylstyrene) ranging in molecular weight from 69000 to 1740000 . The temperature dependence of the tensile creep compliance was described by the method of reduced variables, and the shift factors were identical for all samples, except for those on the level of glassy hardness. The steady-state compliance and tensile viscosity were determined by using the extrapolation method of Ninomiya. It was shown that the dependence of these quantities on molecular weight is much different from that predicted by the Rouse-Bueche theory or by the semi-empirical Ferry-Landel-Williams theory. That is, in the region of molecular weight somewhat above the entanglement region the steady-state compliance is independent of molecular weight. At lower molecular weights the values of the steady-state compliance increase with decreasing molecular weight. The dependence of tensile viscosity on molecular weight follows the familiar 3.4 power law up to molecular weight of about $2 \times 10^{5}$, but there is a distinct decrease in the viscosity dependence upon molecular weight at higher molecular weights. The value of the average molecular weight between entanglements was derived from tensile creep compliance versus temperature curves by use of a reference value of time equal to $1000 \mathrm{sec}$. The value evaluated decreased with increasing molecular weight up to about $2 \times 10^{5}$ and then leveled off to a certain constant value.

KEY WORDS Viscoelasticity/Monodisperse Polymers/Tensile Creep/ Poly( $\alpha$-Methylstyrene) / Compliance / Tensile Viscosity/Molecular Weight / Temperature / Entanglement /
\end{abstract}

The theory of Rouse and Bueche ${ }^{1,2}$ is highly successful in explaining viscoelastic properties of dilute polymer solutions on the molecular level. At present the theory is believed to work quite well also for low molecular weight bulk (undiluted) polymers where entanglement coupling is absent. However, attempts to describe the viscoelastic behavior of bulk polymers of high molecular weight have not been successful. An extension of the theory to bulk polymers of high molecular weight was made by Ferry, Landel, and Williams ${ }^{3}$ shortly after the publication of the Rouse and Bueche papers. They took into consideration the phenomenon of entanglement coupling and modified the RouseBueche theory by introducing certain empirical factors. For some bulk polymer systems the Ferry-Landel-Williams modification has been able to satisfactorily explain observed facts, and the modification has been almost universally accepted. However, for a majority of available experimental data molecular weight distributions of the sample have not been sharp but fairly broad even when the sample had been carefully fractionated by using conventional precipitation techniques. Therefore, until a special synthetic technique for obtaining polymers of very sharp molecular weight distribution became available, 
there was little alternative, in the absence of precise knowledge of the molecular weight distribution, but to rely on comparing the experimental viscoelastic functions with theoretical predictions for a homogeneous (monodisperse) polymer and seeing if the discrepancies were qualitatively in line with results of theoretical treatments concerning the effect of the molecular weight distribution.

Recently, by using five anionically polymerized samples of polystyrene ranging in molecular weight from 80000 to 270000 , Tobolsky, Aklonis, and Akovali ${ }^{4}$ examined critically the predictions of the Rouse-Bueche theory and its modification by Ferry, Landel, and Williams. They found that the dependence on molecular weight of the experimental viscoelastic functions evaluated from torsional creep and stress relaxation measurements was quite different from that predicted by the Rouse-Bueche theory or by the Ferry-Landel-Williams modification. For instance, the observed values of the tensile viscosity $\eta_{l}$ had a fourth power dependence on molecular weight, and those of the steady-state shear compliance $J_{e}$ were found to be nearly independent of molecular weight. According to the RouseBueche theory both $\eta_{l}$ and $J_{e}$ should vary as the first power of molecular weight. The prediction of the Ferry-Landel-Williams theory for $J_{e}$ is the same as that of the Rouse-Bueche theory, but for $\eta_{l}$ the former theory predicts the exponent unity is replaced by 3.4. An extension of the results of Tobolsky et al. was made by Akovali at a molecular weight range up to 1320000 and a similar dependence of these quantities on molecular weight was found. ${ }^{5}$

Somewhat contradictory results were reported by Ninomiya and Ferry on the molecular weight dependence of experimental viscoelastic functions of polyvinyl acetate. ${ }^{6}$ They made the tensile creep and shear creep measurements on sharp fractions ranging in molecular weight from 5500 to 780000 . Their results demonstrated that values of the shear viscosity $\eta$ followed the familiar 3.4 power law and those of the steady-state shear compliance $J_{e}$, unlike the results of Tobolsky et al., varied as the first power of molecular weight. All these experimental facts show that the viscoelastic behavior of sharp fractions of polyvinyl acetate agrees well with the prediction of the
Ferry-Landel-Williams theory.

Concerning the molecular weight dependence of $J_{e}$, very recent observations by Fujimoto, Ozaki, and Nagasawa on monodisperse $\operatorname{poly}(\alpha-$ methylstyrene $)^{7}$ appear to be situated midway between the results of Akovali and those of Ninomiya and Ferry. They studied the tensile stress relaxation behavior of samples of molecular weights ranged from 40000 to 500000 . In the region of low molecular weight $\left(<10^{5}\right)$ the observed values of $J_{e}$ varied as the first power of the molecular weight. However, at medium and high molecular weights values of $J_{e}$ showed distinctly negative deviations, and the deviations increased with increasing molecular weight. On the other hand they showed that values of $\eta$ followed the 3.4 power dependence on molecular weight.

These differences in observed facts might arise to some extent from a variation in the chemical structural factors of the polymer concerned. More subtle differences in molecular weight distribution of samples would be also an important factor which should be considered more carefully before drawing any conclusion. However the available data which are concerned with the viscoelastic behavior of bulk polymers of narrow molecular weight distribution are as yet limited to only few systems, and it appears that more experimental material is needed for establishing the observed facts.

We here present some tensile creep measurements on monodisperse $\operatorname{poly}(\alpha$-methylstyrene $)$ ranging in molecular weight from 69000 to 1740000. These were undertaken with the main aim of establishing, for bulk polymers having very narrow distributions, the relation between molecular weight and the steady-state tensile creep compliance $D_{e}$ or the tensile viscosity $\eta_{l}$ over a wide range of molecular weights. $\operatorname{Poly}(\alpha-$ methylstyrene) has the advantage that by using the anionic polymerization technique followed by column fractionation it may be easily prepared as an essentially monodisperse linear material. Creep measurements provide a direct method of determining the values of $D_{e}$ and $\eta_{l}$, these values being more reliably obtained from creep measurements than from stress relaxation. Similar work on monodisperse poly(cis-1, 4isoprene) and polystyrene will be reported 
subsequently.

\section{EXPERIMENTAL}

\section{Materials}

The poly $(\alpha$-methylstyrene) studied was prepared by anionic polymerization in vacuo at $-78^{\circ} \mathrm{C}$ with $n$-butyl-lithium as the initiator and tetrahydrofuran as the solvent. The apparatus and technique used for the preparation were similar to those described by Morton et al. ${ }^{8}$ and by Fujimoto et al. ${ }^{9} \quad$ A possible tail in the molecular weight distribution in the lowest end was eliminated by fractionation at $34.5^{\circ} \mathrm{C}$ using a large scale elution column with benzene-ethyl alcohol mixtures. The number-average molecular weight, $M_{n}$, was determined by osmometry in toluene at $37^{\circ} \mathrm{C}$ using a high-speed membrane osmometer (Mechrolab Model 502). The weight-average molecular weight, $M_{w}$, was evaluated from intrinsic viscosity using an empirical relation for the monodisperse polymer, $[\eta]=1.18 \times 10^{-4} \times$ $M_{w}{ }^{0.70}$ (toluene, $25^{\circ} \mathrm{C}$ ). ${ }^{10}$ Viscosity measurements were carried out by using a dilution viscometer of the Ubbelohde type. The number-average and weight-average molecular weights of samples are given in Table I. The sedimentation boundary curve of each sample was also obtained by a Spinco-E ultracentrifuge at the theta point (cyclohexane, $39^{\circ} \mathrm{C}$ ), and the ratio $M_{w} / M_{n}$ was evaluated thetefrom. The results are also given in the fourth column of Table I.

Table I. Monodisperse poly $(\alpha$-methylstyrene)

\begin{tabular}{rccc}
\hline Sample & $M_{w}$ & $M_{n}$ & $M_{w} / M_{n}$ \\
\hline MS-1 & $1.74 \times 10^{6}$ & $\ldots$ & 1.00 \\
2 & $1.89 \times 10^{5}$ & $1.87 \times 10^{5}$ & 1.01 \\
3 & $2.29 \times 10^{5}$ & $2.27 \times 10^{5}$ & 1.01 \\
4 & $1.05 \times 10^{5}$ & $1.02 \times 10^{5}$ & 1.02 \\
5 & $1.23 \times 10^{5}$ & $1.51 \times 10^{5 a}$ & 1.02 \\
6 & $3.86 \times 10^{5}$ & $4.03 \times 10^{5}$ & 1.01 \\
7 & $6.90 \times 10^{4}$ & $5.74 \times 10^{4 a}$ & $\ldots$
\end{tabular}

a The determination was somewhat less accurate compared with the other samples.

For tensile creep measurements, thin films were obtained by slow evaporation of solvent from a $10 \%$ methylene chloride solution on a clean mercury surface. Final traces of the solvent were removed by prolonged drying in a vacuum oven at $180^{\circ} \mathrm{C}$. The weight of films was checked to insure the complete volatization of methylene chloride. Usually it took about three weeks or more to complete the drying process. Under such drying conditions thermal degradation of samples does not take place to any extent. The film thickness was measured by means of a micrometer with a precision of about $\pm 2 \times 10^{-4} \mathrm{~cm}$, and both the length and width were measured with a precision of about $\pm 0.02 \mathrm{~cm}$. The films used for tensile creep measurements were about $3 \times 10^{-2} \mathrm{~cm}$ thick, $1 \mathrm{~cm}$ wide, and $2 \mathrm{~cm}$ long.

\section{Method}

The tensile creep measurements were made with a creep balance type apparatus. The essential part of the balance consisted of a vertical brass post, near the top of which was pivoted an aluminum alloy pulley. Over the pulley was hung a fine copper wire, at one end of which was attached the upper clamp for the sample film and at the other an iron core of a linear variable differential transformer plus a loading weight. The lower clamp for the sample film mounting was fixed to a base platform. The apparatus and procedure have been described in detail elsewhere. ${ }^{11}$ The change in length of the sample film was detected by a linear variable differential transformer, and was amplified and recorded by means of a recorder (Iwamoto Seisakusho Ltd., Kyoto). With these arrangements a change in length of $5 \times 10^{-4} \mathrm{~cm}$ could be measured with a precision of about $\pm 2 \times$ $10^{-4} \mathrm{~cm}$. Thus, with the use of films of length about $2 \mathrm{~cm}$, our resolution in length corresponds to a tensile creep compliance of about $1 \times$ $10^{-10} \mathrm{~cm}^{2} /$ dyn.

Measurements were performed from 140 to $210^{\circ} \mathrm{C}$ except for sample MS- 1 (the highest molecular weight sample). For sample MS-1, in order to obtain creep behavior in the terminal zone, measurements were extended up to $230^{\circ} \mathrm{C}$, though the measuring period at these higher temperatures was limited to about 20 minutes to avoid thermal degradation of the sample. The temperature at any point near the sample film was kept constant with a precision of about $\pm 0.1^{\circ} \mathrm{C}$ between 140 and $220^{\circ} \mathrm{C}$, and within $\pm 0.3^{\circ} \mathrm{C}$ at $230^{\circ} \mathrm{C}$. 
It has been reported that molecular degradation of $\operatorname{poly}(\alpha$-methylstyrene $)$ took place to some extent during a one hour treatment in the temperature region above $220^{\circ} \mathrm{C} .{ }^{12 a, b}$ The thermal degradation of samples after the tensile creep measurements in the region of higher temperatures was examined for samples MS- 1 and -6 from viscosity measurements in toluene at $25^{\circ} \mathrm{C}$. For the latter sample no degradation was confirmed after about three hours of creep measurements at $200^{\circ} \mathrm{C}$. However for the former, the molecular weight of the sample, evaluated from intrinsic viscosity, decreased to $1.4 \times 10^{6}$ after the same treatment. It was also found for sample MS-1 that the decrease in molecular weight by treatment at $230^{\circ} \mathrm{C}$ was of the same order as described above after about half an hour. Accordingly, measurements at higher temperatures were limited to an interval in which the thermal degradation did not take place to any extent. Fujimoto et al. observed that no thermal degradation of the polymer occurred after stress relaxation measurements at $220^{\circ} \mathrm{C} .^{7}$

In all cases the maximum tensile strain including viscous flow did not exceed $20 \%$ and was much less than this at lower temperatures.

\section{RESULTS AND DISCUSSION}

The tensile creep compliance $D_{p}(t)$ of sample MS-3 is plotted logarithmically against time $t$ in Figure 1 at ten temperatures from 148 to $212^{\circ} \mathrm{C}$. Here the subscript $p$ denotes that $D(t)$ has been multiplied by $T \rho / T_{0} \rho_{0}$, where $\rho$ and $\rho_{0}$ are the densities at temperatures $T$ and $T_{0}$, and $T_{0}$ is a reference temperature. The reference temperature was chosen as $172^{\circ} \mathrm{C}$. The vertical adjustment $T \rho / T_{0} \rho_{0}$ permits temperature shift factors to be determined from horizontal displacements of $D_{p}(t)$ curves at different temperatures. Similar families of creep curves were obtained for other samples.

Figure 2 shows the master curves which were obtained by shifting the experimental curves according to the time temperature superposition principle. The many individual points for different temperatures, not shown except for sample MS-3, lay mostly within $2 \%$ of the master curves. The shift factor $a_{T}$ used for the reduction is plotted semilogarithmically against $T-T_{0}$

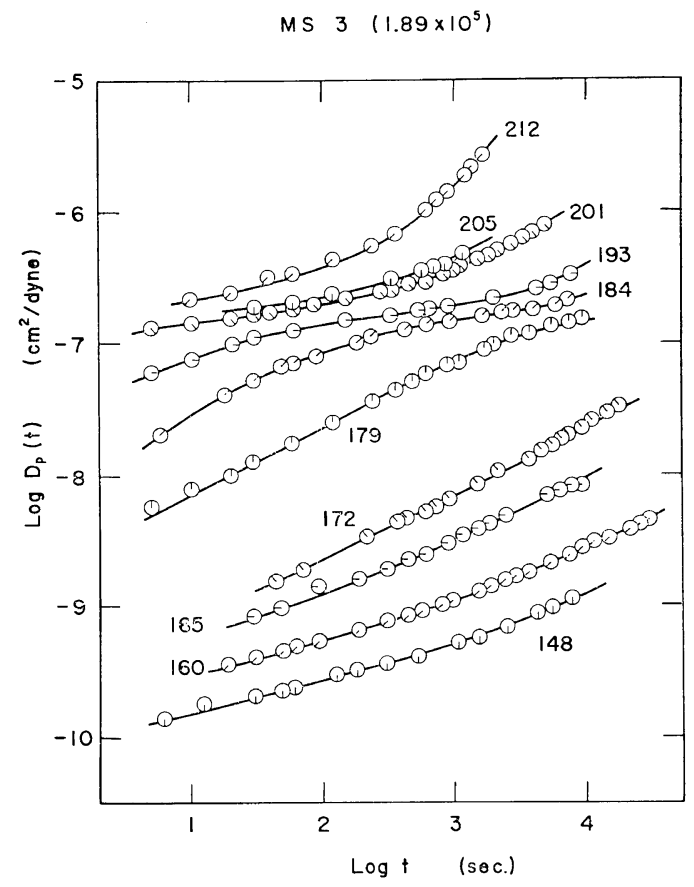

Figure 1. Tensile creep compliance of sample MS-3, plotted logarithmically against time at ten temperatures as indicated.

in Figure 3. It is seen that a single composite curve can be obtained for all samples at temperatures above the reference temperature. This means, in this temperature region, that over a fairly wide range of molecular weights the temperature dependence of viscoelastic behavior of monodisperse poly $(\alpha$-methylstyrene $)$ can be described by the WLF equation ${ }^{13}$

$$
\log a_{T}=-C_{1}\left(T-T_{0}\right) /\left(C_{2}+T-T_{0}\right)
$$

with a single set of parameters which in turn are related to free volume parameters. The solid curve in Figure 3 corresponds to the WLF equation with $T_{0}=172^{\circ} \mathrm{C}, C_{1}=13.7$, and $C_{2}=$ 49.3. From these WLF parameters we obtain $f_{172}=0.032$ for the fractional free volume at $172^{\circ} \mathrm{C}$ and $\alpha=6.4 \times 10^{-4}$ for the thermal expansion coefficient of the free volume. According to our recent dilatometric measurements on monodisperse $\operatorname{poly}(\alpha$-methylstyrene $)$, the glass transition temperature is practically the same for all samples studied here and is in the vicinity of $167^{\circ} \mathrm{C}^{14}$ The corresponding free volume at the glass transition temperature is 0.029 . In the 


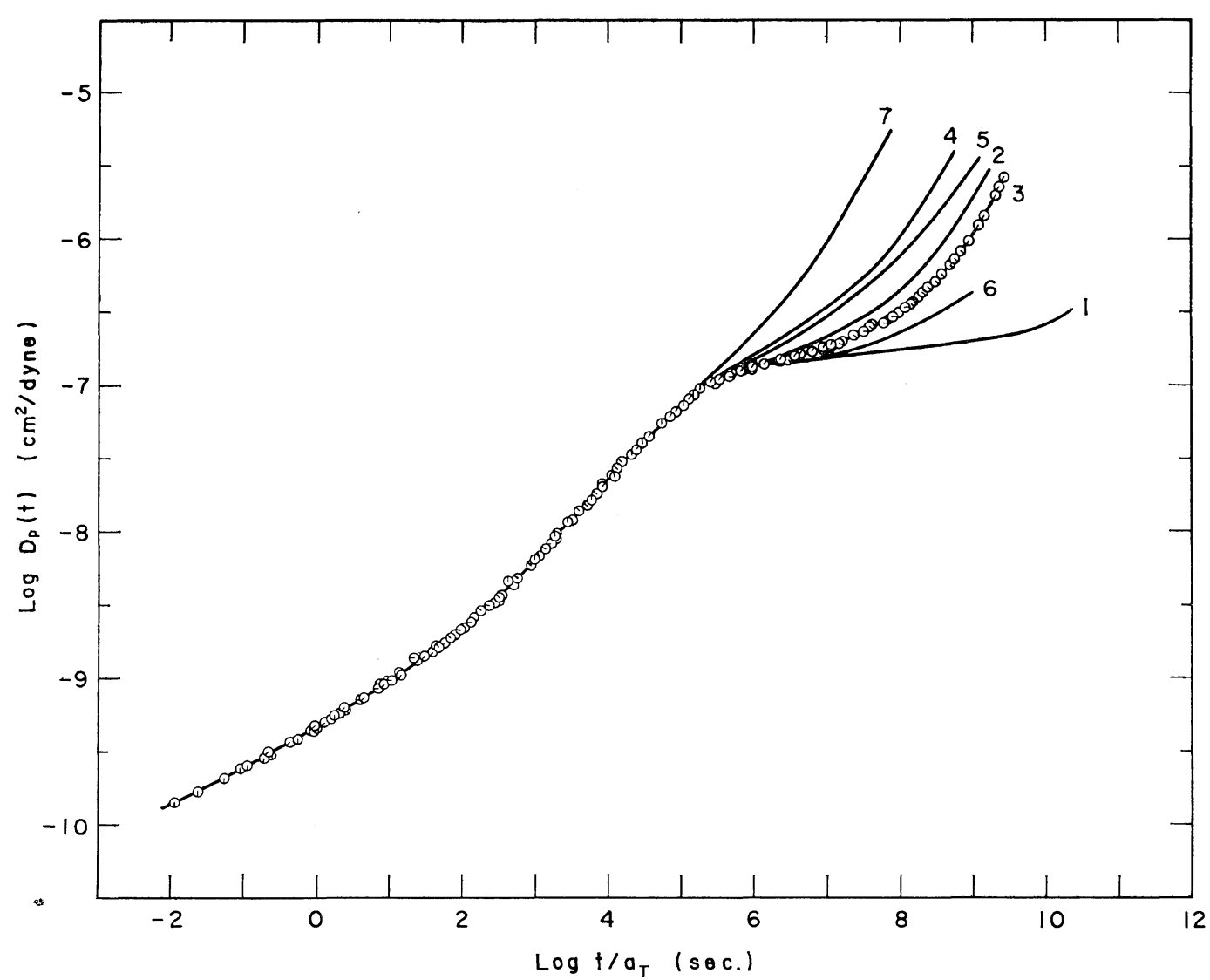

Figure 2. Tensile creep master curves for seven poly $(\alpha$-methylstyrene $)$ samples as indicated at $172^{\circ} \mathrm{C}$.

temperature region below the reference temperature the divergence of the plots is seen in Figure 3. One factor which could possibly account for this divergence might arise from the uncertainty associated with the complete removal of residual stresses in the annealing of samples. The somewhat lower accuracy in making measurements on this level of glassy hardness could also be responsible for the divergence noted. More accurate measurements in the glassy and transition regions of monodisperse polymer solids are now being carried out in our laboratory by means of a torsional creep apparatus.

The steady-state compliance $D_{e}$ and the tensile viscosity $\eta_{l}$ were determined by using the extrapolation method of Ninomiya. ${ }^{15}$ That is, a plot of $m D(t) / t v s \cdot 1 / t$ yields a relatively linear extrapolation to the intercept and this intercept gives the value of $1 / \eta_{l}$, where $m$ denotes $d \log$ $D(t) / d \log t$. Alternatively, the reciprocal of $\eta_{l}$ can be evaluated from the intercept of a plot of $D(t) / t v s .1 / t$, and the value of $D_{e}$ from the initial tangent of this plot. Figure 4 shows the plots of $D(t) / t$ and $m D(t) / t$ vs. $1 / t$ for the sample MS-2 at $200^{\circ} \mathrm{C}$. It can be seen from the figure that the plot of $m D(t) / t v s$. $1 / t$ can be approximated by a straight line over a fairly long period of time. The values of $D_{e}$ and $\eta_{l}$ obtained at $200^{\circ} \mathrm{C}$ by this method are given in Table II.

In the case of the highest molecular weight sample MS-1, as previously mentioned the length of time over which tensile creep measurements at higher temperatures were made had to be kept short since unfavorable thermal degradation of the polymer began occurring after half an hour. Accordingly, in order to extend the creep curve at $200^{\circ} \mathrm{C}$ from the plateau region to the 


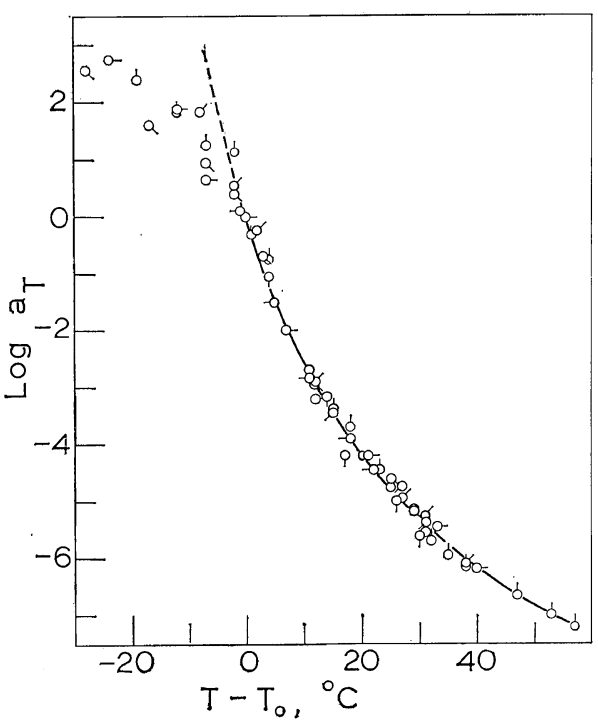

Figure 3. Log $a_{T}$ plotted against $T-T_{0}$ for seven poly $(\alpha$-methylstyrene) samples.

oे, MS-1; o, MS-2; 0-, MS-3; Q, MS-4;

१, MS-5; ○, MS-6; -0 , MS-7.

Table II. Steady-state compliances and tensile viscosities of seven monodisperse $\operatorname{poly}\left(\alpha\right.$-methylstyrene)s at $200^{\circ} \mathrm{C}$

\begin{tabular}{rll}
\hline Sample & $\begin{array}{c}D_{e} \\
\left(\mathrm{~cm}^{2} / \mathrm{dyn}\right)\end{array}$ & $\begin{array}{c}\eta_{l} \\
\text { (poise) }\end{array}$ \\
\hline MS-1 & $1.88 \times 10^{-7}$ & $1.89 \times 10^{12}$ \\
2 & $4.20 \times 10^{-7}$ & $7.05 \times 10^{9}$ \\
3 & $2.64 \times 10^{-7}$ & $1.43 \times 10^{10}$ \\
4 & $8.69 \times 10^{-7}$ & $1.95 \times 10^{9}$ \\
5 & $7.23 \times 10^{-7}$ & $4.00 \times 10^{9}$ \\
6 & $2.27 \times 10^{-7}$ & $4.17 \times 10^{10}$ \\
7 & $3.90 \times 10^{-6}$ & $2.04 \times 10^{8}$ \\
\hline
\end{tabular}

terminal zone, creep curves which were obtained at above $200^{\circ} \mathrm{C}$ were grafted on the curve at $200^{\circ} \mathrm{C}$, according to the method of reduced variables, and the extrapolation method was applied to the composite curve. Therefore, the determination may be less accurate compared with that for other lower molecular weight samples. In addition, in the case of a high molecular weight sample, such as MS-1 and -6 , it may be possible that the determination of $D_{e}$ and $\eta_{l}$ involves another factor of uncertainty because the extrapolation to infinite time was made from the region where the values of $m$ were about 0.3 .

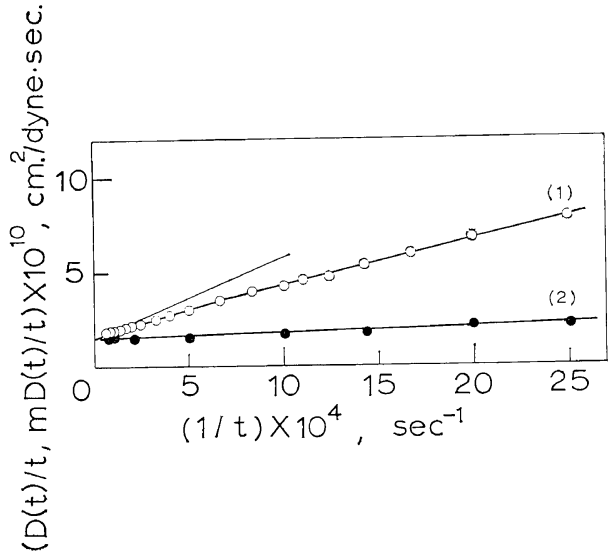

Figure 4. $D(t) / t$ (curve 1) and $m D(t) / t$ (curve 2) of sample MS-2 plotted against $1 / t$ at $200^{\circ} \mathrm{C}$.

In such a region of relatively small $m$ it seems doubtful whether the true steady-state flow is reached or not. However, as seen in Figure 4, the plots of $m D(t) / t$ vs. $1 / t$ for samples of relatively lower molecular weight are practically linear over the range of $0.2<m<0.9$, and their slopes are rather low. This enables us to estimate the intercept of the $m D(t) / t v s$. $1 / t$ plot with reasonable accuracy. Thus, even when the molecular weight of a sample is relatively high, the extrapolation method can be expected to provide a reasonable determination of $D_{e}$ and $\eta_{l}$, though not an extremely accurate one.

In Figure 5 the values of $D_{e}$ determined at $200^{\circ} \mathrm{C}$ are plotted logarithmically against $M_{w}$. It can be seen that the values of $D_{e}$ decrease

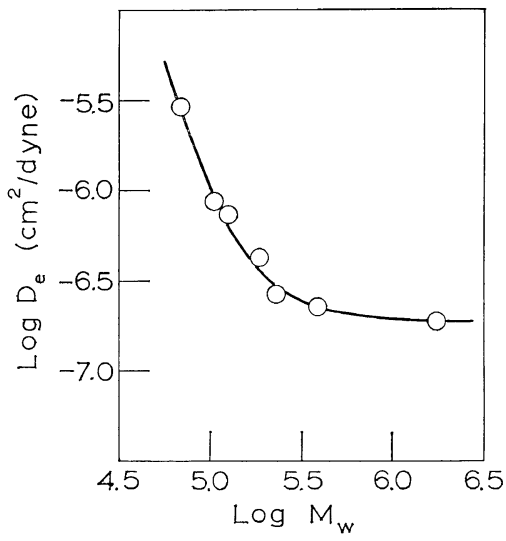

Figure 7. $M_{e}$ plotted against $\log M_{w}$ for six poly $(\alpha$-methylstyrene) samples. 
with increase of molecular weight and are nearly independent of molecular weight in the region of higher molecular weights. These are in contrast to the prediction of the Rouse-Bueche theory $^{1,2}$ and also that of the Ferry-LandelWilliams theory: ${ }^{3}$ they both predict that steadystate compliance should be proportional to molecular weight. It was predicted theoretically, and also shown experimentally, that steady-state compliance is highly sensitive to the polydispersity of the polymer samples, and increases with the breadth of molecular weight distribution. ${ }^{16}$ As shown in Table I, the molecular weight distributions of our samples of lower molecular weights are unfortunately less sharp compared with those of higher molecular weights. However it is our belief that, in the region of relatively low molecular weight, this may not be the conclusive reason for the observed fact, since even for the lowest molecular weight sample the molecular weight distribution is evidently too sharp to explain the positive deviation of the plot by the effect of polydispersity of the sample. The molecular mechanism underlying this feature is not clearly understood at present but it is feasible that it could be closely connected with the phenomenon of entanglement coupling, which plays an important role in viscoelastic properties of polymers of molecular weight above a certain critical value, and also with the molecular conformation of polymer molecules of rather low molecular weight in the bulk state. In the region of higher molecular weight, above about $2 \times 10^{5}$, the values of $D_{e}$ are nearly constant. It should be emphasized that, in this region of molecular weight, the quite similar independence of the steady-state compliance on molecular weight is observed for monodisperse polystyrene $e^{4,5}$ and poly(cis-1,4-isoprene). ${ }^{17}$ As mentioned before, results of Fujimoto et al., ${ }^{7}$ which were obtained from stress relaxation studies of monodisperse poly $(\alpha$-methylstyrene), do not agree completely with these facts. However, their data show distinctly that the molecular weight dependence of the steady-state compliance decreases with an increase of molecular weight. Thus, in the region of rather higher molecular weights it appears that the independence of the steadystate compliance on molecular weight is one of the characteristic features of monodisperse poly-

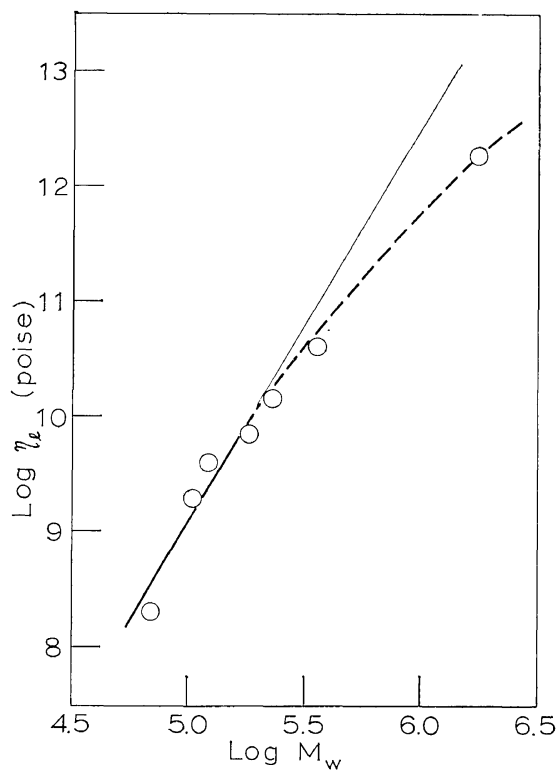

Figure 6. $\eta_{l}$ at $200^{\circ} \mathrm{C}$ plotted logarithmically against $M_{w}$ for seven $\operatorname{poly}(\alpha$-methylstyrene) samples.

mers in the bulk state, though at present data are limited to only a few systems.

The values of $\eta_{l}$ at $200^{\circ} \mathrm{C}$ are plotted logarithmically against $M_{w}$ in Figure 6 . It can be seen that plots can be represented by a straight line for molecular weights lower than about $2 \times 10^{5}$. The slope of the straight line is 3.4. An interesting and rather surprising consequence is that plots in the region of molecular weight greater than about $2 \times 10^{5}$ are not fitted by the straight line but a curve concave to the abscissa. The Rouse-Bueche theory predicts that the viscosity should be proportional to molecular weight, and the Ferry-Landel-Williams modification predicts thas $\eta_{l}$ should vary as the 3.4 power of molecular weight. As described before, for the highest molecular weight sample, MS-1, the determination of $\eta_{l}$ involves a less direct calculation and there is also a possibility that the sample has been subjected to thermal degradation during the course of the tensile creep measurements, though it is considered to be of very slight extent. Even though this is taken into consideration, in Figure 6 the negative deviations from the familiar 3.4 power law are still apparent at molecular weights higher than about $2 \times 10^{5}$. The results cannot be accounted 
for by assuming some branching for high molecular weight samples. Such a contribution is expected to show more pronounced negative deviations. At the moment, the molecular mechanism underlying the feature is not known to us, but is vaguely conceived as a result of a rather compactly coiled structure of the individual polymer molecule in the solid state, which is unfavorable to the formation of entanglement coupling with other molecules. There is the possibility that certain preparation procedures, such as the choice of casting solvent and drying and/or annealing treatments, may have an important influence on the structure of the polymer chain, unless they are carried out at temperature fairly well above the glass transition temperature of the polymer. In this sense our experimental findings may still be ambiguous because the rather low thermal stability of $\operatorname{poly}(\alpha-$ methylstyrene) is a serious obstacle to various treatments of the sample at higher temperatures. More elaborate studies, which are aimed at obtaining clearer understanding of the internal structure of monodisperse polymer solids by using thermally stable polymers, may be able to elucidate the observed fact. Concerning the dependence of viscosity on molecular weight it should be noted that Tobolsky et al. ${ }^{4}$ have discussed a complex feature of the plot of $\log$ viscosity versus log molecular weight for polystyrene above the entanglement region. That is, slightly above the entanglement region the slope of the plot is 3.4; at a molecular weight of about $2 \times 10^{5}$ the slope has increased to 4.0 , and in the very high molecular weight range, about $10^{6}$, there is a distinct tendency for the plot to level off. At any rate, though at present there is some divergence among

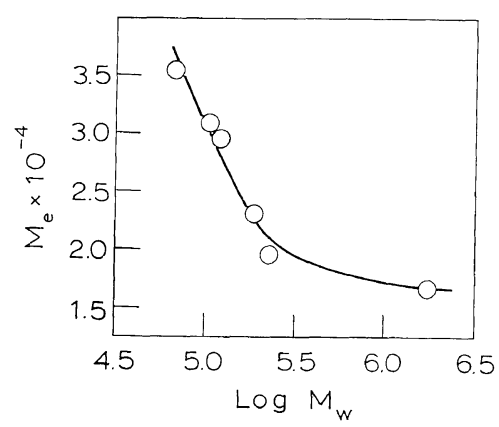

Figure 5. $D_{e}$ at $200^{\circ} \mathrm{C}$ plotted logarithmically against $M_{w}$ for seven $\operatorname{poly}(\alpha$-methylstyrene) samples. the experimental findings, it may be concluded that above the entanglement region, except in the very high molecular weight range, the viscosity of monodisperse polymers varies as 3-4 power of molecular weight.

Recently Tobolsky and Takahashi ${ }^{18}$ have estimated the value of the average molecular weight between entanglements, $M_{e}$, from shear modulus measurements at a fixed time as a function of temperature. They calculated $M_{e}$ from the characteristic parameter $E_{2}$ by using the theory of rubber elasticity. Here $E_{2}$ is the value of $3 G(10)$, the rigidity modulus at $10 \mathrm{sec}$ multiplied by three, which is selected at the point in the rubbery plateau region where the slope of $\log [3 G(10)] v s$. temperature is at a minimum. In order to estimate the value of $M_{e}$ for our monodisperse $\operatorname{poly}(\alpha$-methylstyrene)s, we may apply a quite similar procedure to the plots of the $1000 \mathrm{sec}$ tensile creep compliance, $D(1000)$, against temperature. A characteristic parameter $D_{2}$ may then be determined as the value of $D(1000)$ at the inflection in the plateau region of $\log D(1000)$ versus temperature plots. We here chose $1000 \mathrm{sec}$ as a fixed time. From the value of $D_{2}$ thus obtained, the average molecular weight between entanglements is calculated from the equation

$$
M_{e}=3 d R T_{1} D_{2}
$$

where $d$ is the density, $R$ is the gas constant, and $T_{1}$ is the absolute temperature at which $D_{2}$ is determined.

Figure 7 gives plots of $M_{e} v s . \log M_{w}$. The shape of the plots closely resembles that of logarithmic plots of $D_{e}$ versus $M_{w}$ shown in Figure 5. The values of $M_{e}$ decrease with an increase of $M_{w}$ up to about $2 \times 10^{5}$ and then level off to a certain constant value. Since the molecular weight of the lowest molecular weight sample is comparable to that between entanglements, the former feature may tentatively be interpreted as being due to contribution from free chain ends. For the latter feature, it appears that the formation of an entanglement network would be responsible. However, data available at the present time are so limited that more studies will have to be carried out before any theoretical interpretation of the observed facts can be attempted. 


\section{Concluding Remarks}

Summarizing the experimental findings not only for $\operatorname{poly}(\alpha$-methylstyrene) reported here but also for other polymers, we may accept the following as a general conclusion for the experimentally observed viscoelastic properties of monodisperse polymer solids. Over the relatively wide range of molecular weights from somewhat above the entanglement region to a molecular weight of about $1 \times 10^{6}$, steady-state compliance is independent of molecular weight and the viscosity depends on molecular weight the values of the power index ranging from 3 to 4 . Unfortunately, at the moment, none of the currently accepted molecular theories can satisfactorily explain the experimental findings. It may be concluded that only those molecular theories which take into consideration the effect of molecular entanglement in a more elaborate manner will be able to elucidate the observed facts.

Acknowledgements. The authors wish to express their appreciation for the many useful discussions held with the late Dr. Masaaki Fukatsu of this University. We are also indebted to Mr. Mitsuo Abe of the Japan Synthetic Rubber Co., Ltd., for measurements of number average molecular weight, and to $\mathrm{Mr}$. Nobu Kitamura for his help in viscosity measurements.

\section{REFERENCES}

1. P. E. Rouse, J. Chem. Phys., 21, 1272 (1953).

2. F. Bueche, J. Chem. Phys., 22, 603 (1954).
3. J. D. Ferry, R. L. Landel, and M. L. Williams, J. Appl. Phys., 26, 359 (1955).

4. A. V. Tobolsky, J. J. Aklonis, and G. Akovali, J. Chem. Phys., 42, 723 (1965).

5. G. Akovali, J. Polym. Sci., Part A-2, 5, 875 (1967).

6. K. Ninomiya and J. D. Ferry, J. Phys. Chem., 67, 2292 (1963).

7. T. Fujimoto, N. Ozaki, and M. Nagasawa, $J$. Polym. Sci., Part A-2, 6, 129 (1968).

8. M. Morton, A. A. Rembaum, and J. L. Hall. J. Polym, Sci., Part A, 1, 461 (1963).

9. T. Fujimoto, N. Ozaki, and M. Nagasawa, $J$. Polym. Sci., Part A, 3, 2259 (1965).

10. T. Fujimoto and M. Nagasawa, unpublished results.

11. H. Odani, S. Kitamura, N. Nemoto, and M. Kurata, Bull. Inst. Chem. Res. Kyoto Univ., 45, 122 (1967).

12. a) J. M. G. Cowie and S. Bywater, J. Polym. Sci., 54, 221 (1961);

b) D. Braun and G. Heufer, Makromol. Chem., 79, 98 (1964).

13. M. L. Williams, R. F. Landel, and J. D. Ferry, J. Amer. Chem. Soc., 77, 3701 (1955).

14. N. Nemoto, K. Nishida, H. Odani, and $M$. Kurata, in preparation.

15. K. Ninomiya, J. Phys. Chem., 67, 1152 (1963).

16. For instance, J. D. Ferry, "Viscoelastic Properties of Polymers," John Wiley and Sons, Inc., New York, N. Y., 1961, p. 171.

17. N. Nemoto, M. Moriwaki, M. Matsubara, H. Odani, and M. Kurata, unpublished results.

18. A. V. Tobolsky and M. Takahashi, J. Appl. Polym. Sci., 7, 1341 (1963). 